\title{
Nipple Skin Trauma in Breastfeeding Women During Postpartum Week One
}

\author{
Maya Nakamura, Yoko Asaka,, Tomoko Ogawara, and Yutaka Yorozu²
}

\begin{abstract}
Objective: To objectively describe changes to nipple skin and classify signs of nipple trauma in breastfeeding women during postpartum week 1.

Methods: This study was conducted in two phases. Phase 1 was an observational prospective study of breastfeeding mothers in which data were obtained from photographs and digital images of nipple skin and analyzed to anatomically classify signs of nipple trauma. In Phase 2, the reliability of signs identified in Phase 1 was verified with the cooperation of eight clinical midwives.

Results: A total of 776 images of 50 breastfeeding mothers were obtained daily. The signs of nipple trauma included erythema, swelling, blistering, fissure, and scabbing. Purpura and peeling were identified only with photographic image analysis. Scabbing and blistering were classified as damage to the dermis, and erythema and swelling as damage to the epidermis, based on anatomical evidence and the mothers' subjective experiences of pain intensity. Erythema and swelling were observed from day 0, with erythema most frequently observed. For inter-rater reliability of the five signs of nipple trauma, Kendall's coefficient of concordance ranged from 0.46 (moderate) to 0.85 (almost perfect). Reliability was high for fissure, substantial for blistering and scabbing, and moderate for erythema and swelling.

Conclusions: Image analysis revealed five signs of nipple trauma. Erythema and swelling were the most frequently observed signs during postpartum week 1 . However, the agreement rate was lower than that for other signs, suggesting the possibility of conflicting interpretations in clinical evaluation.
\end{abstract}

Keywords: nipple trauma, breastfeeding, image analysis

\section{Introduction}

$\mathbf{N}$ IPPLE TRAUMA IS a major reason why women abandon breastfeeding. ${ }^{1,2}$ Previous studies have revealed that nipple trauma occurs in $29-76 \%$ of breastfeeding mothers within postpartum week $1 .^{3}$ Nipple trauma causes pain and discomfort, which render it difficult for the mother to continue breastfeeding. Therefore, prevention and treatment of nipple trauma during the early postpartum period are essential for successful breastfeeding.

Over the years, various methods have been used for prevention and treatment of nipple trauma, and although several studies have been conducted to validate the effectiveness of these methods, ${ }^{3-9}$ there is no consensus on the most effective strategies. Some methodological issues have been addressed, such as the small number of subjects ${ }^{9}$ as well as the lack of a standardized definition of nipple trauma. ${ }^{10}$
Previous studies have stated that evaluation of nipple trauma can lead to conflicting interpretations because of the lack of a standardized definition of nipple trauma. ${ }^{10}$ This lack of standardization has led to discrepancies in the nomenclature of nipple lesions among published articles, and conflicting interpretations exist in the assessment of the severity of nipple trauma. ${ }^{10}$ Thus, expanding the understanding of this phenomenon and its healing process is necessary to improve prevention and treatment of nipple trauma, and to achieve this goal, a standardized classification of nipple trauma is required. Therefore, this study was aimed at describing changes to nipple skin and classifying the signs of nipple trauma in breastfeeding women during postpartum week 1.

This study was conducted in two phases. Phase 1 was an observational prospective study of breastfeeding mothers, and based on the results of Phase 1, the reliability of signs of nipple trauma was validated in Phase 2.

\footnotetext{
${ }^{1}$ Faculty of Health Sciences, Hokkaido University, Sapporo, Japan.

${ }^{2}$ Kiyota Women's Clinic, Sapporo, Japan.
} 


\section{Phase 1}

Purpose

To describe changes to the nipple skin and classify signs of nipple trauma in breastfeeding women.

\section{Materials and Methods}

Participants. A total of 50 Japanese women aged $>20$ years were recruited from an obstetrics and gynecology facility in Sapporo, Japan, from October 2016 to January 2017. One participant dropped out of the study on day 3 because of gastroenteritis symptoms. All participants were hospitalized after giving birth. Each of the women breastfed (on demand) while residing in the same room as their newborns, supported by midwives throughout the hospitalization period. Latch assessment was individually conducted by the midwives at least three times per day with reference to the Breastfeeding Observation Form, ${ }^{11}$ an observational tool for the breastfeeding mother and baby, generally used for latch assessment.

Data collection. Written consent was obtained from all participants of the study. The bilateral nipple skin of participants was photographed every 24 hours until the day of discharge from the clinic. The average study period for each participant was $4.8 \pm 1.4$ days.

Photographs were taken in macro mode using a digital camera (Nikon COOLPIX A 300N 1517; Nikon Co., Tokyo, Japan) under lighting of between 100 and 300 lux. A color chart for image correction $\left(\mathrm{CASMATCH}^{\circledR}\right.$; KENIS Limited Co., Osaka, Japan) was set parallel to the nipple area and frontal views were photographed. When changes to the lateral portion of the nipple were observed, additional shooting angles were added, and data collection was continued. To minimize the effects of breastfeeding, all photographs were taken by the same trained researcher. The photographs were processed using color correction by CASMATCH (KENIS Limited Co.) and size correction was performed using image-editing software (Photoshop 6; Adobe System, Inc., Tokyo, Japan).

The intensity of nipple pain experienced by participants at the beginning of breastfeeding was assessed every 24 hours using a 10-point numeric pain scale, with a range of scores from 1 , indicating no pain, to a maximum of 10 , indicating intense pain.

Data analyses. The processed digital images were visually analyzed. The characteristics of observed changes in the nipple skin were determined and examined with reference to previously defined characteristics. ${ }^{5,10}$ Signs of nipple trauma were then anatomically classified, and the number of signs for each postpartum day was counted. If more than one sign was observed on a nipple, each sign was counted as an independent sign and therefore 776 images were taken for the analyses. Visual analyses were conducted by three midwives, two of whom were International Board-Certified Lactation Consultants. ${ }^{\circledR}$ The relationship between the signs and nipple pain was compared for each nipple, and statistical analysis was performed using the chi-square test, Fisher's exact test, and nonparametric Mann-Whitney $U$ test. The level of statistical significance was set at 0.05 .
Ethics committee approval. The study protocol was approved by the Ethics Review Committee of the Graduate School of Health Sciences, Hokkaido University (Approval number: 16-82), and conducted in accordance with ethical principles of the Declaration of Helsinki for medical research involving human subjects.

\section{Results}

A total of 776 images of 50 breastfeeding mothers were acquired daily. The average gestational age at birth of the infants was $38.9 \pm 1.2$ weeks and the average birth weight was $3225.0 \pm 406 \mathrm{~g}$. Forty-five $(90 \%)$ participants gave birth vaginally. Sixteen $(32 \%)$ infants were male. Thirty-four $(68 \%)$ of the 50 women were multiparas and had breastfeeding experience before their most recent birth. Four $(8 \%)$ nipples were categorized as retracted or inverted. The average number of breastfeeding episodes per 24 hours was $9.4 \pm 4.3$. Twenty-nine $(58 \%)$ women received treatment for nipple trauma during the observation period. Of these, lanolin was applied in $20(40 \%)$ women, breast milk in $8(16 \%)$, and moisturizing oil in $1(2 \%)$.

Changes in nipple tissue after initiating breastfeeding. The most common changes to nipple tissue were purpura, peeling, erythema, swelling, blistering, fissure, and scabbing (Fig. 1). Purpura and peeling, which accounted for the smallest number of changes, were observed only on the tip of the nipple by image analysis and were not reported in the medical records. The incidences of erythema, swelling, blistering, fissure, and scabbing were analyzed to classify signs of nipple trauma.

Erythema was observed as recognizable redness on the tip or lateral part of the nipple. None of the women developed an intradermal hemorrhage, and erythema was also observed with other signs, for example, around scabbing or fissures.

Swelling was observed on the epithelial surface, and when swelling was localized, the boundary line appeared as a crack or fissure. Blistering was defined as a transparent and flaccid skin bulge. Blistering was classified as yellow blisters formed by retention of watery components and bright red blisters formed by retention of blood constituents. Fissure was defined as skin continuity with a cleft or linear tissue loss that was observed only on the side of the nipple or on the nippleareolar junction. Scabbing was defined as formation of a crust of hardened blood and serum over the injured tissue. Scabbing formed by blood appeared red, brown, or black and that formed by interstitial fluid appeared yellow.

The observed changes to the nipple skin during the study period are listed in Table 1. Erythema was the most frequent change and was observed in 499 images. It was observed from day 0 , peaked at $75.2 \%$ on day 3 , and fell to $24.0 \%$ on day 7 . Swelling was also observed from day 0 , but peaked at $47.0 \%$ on day 4 , and fell to $12.5 \%$ on day 7 . Scabbing was observed at $23.9 \%$ from day 1 , but unlike erythema and swelling, the incidence of scabbing did not clearly decrease during the observation period. Blistering of the papilla was the rarest sign of nipple trauma, with no clear trend in the number of observations. Fissures were observed only at nipple-areolar junctions. Multiple signs in one papilla were observed in more than $40.0 \%$ of digital images on each postpartum day. The chi-squared test or Fisher's exact test 


\section{【Erythema】}

A

FIG. 1. Erythema was recognizable redness on the tip or lateral part of the nipple (A) and was also observed where the scabbing was peeled off (B). Swelling was observed on the epithelial surface (C). When swelling was localized, the boundary line appeared as a crack or fissure (D). Blistering was classified as yellow blisters formed by retention of watery components (E) and bright red blisters formed by retention of blood constituents (F). Scabbing formed by blood appeared red, brown, or black (G) and that formed by interstitial fluid appeared yellow (H). Fissure was observed only on the side of the nipple or on the nipple-areolar junction (I), and epithelialization was observed (J). Purpura (K) and Peeling $(\mathbf{L})$ are the small changes observed on the tip of the nipple by image analysis. Color images available online at www.liebertpub.com/bfm

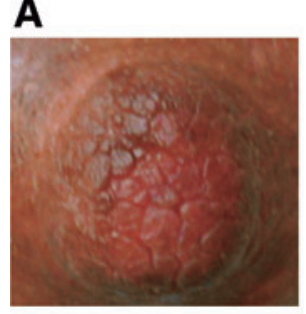

【Blistering】

E

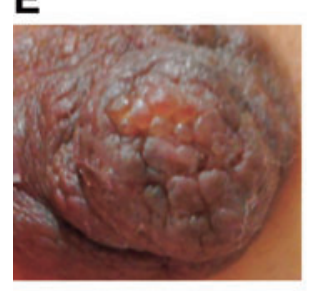

【Fissure】

I

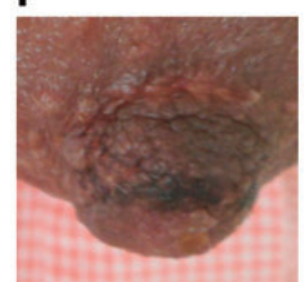

B

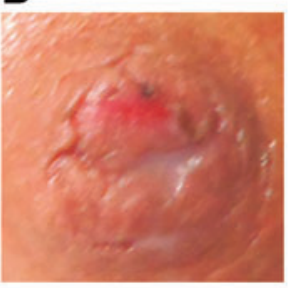

F

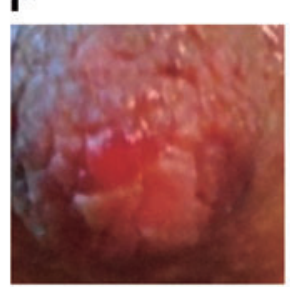

$\mathbf{J}$

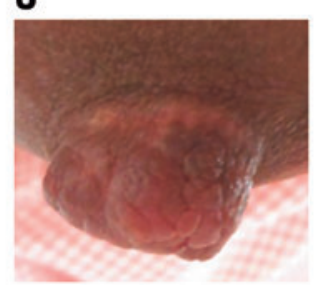

【Swelling】

C

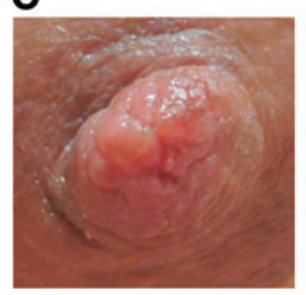

\section{【Scabbing】}

G

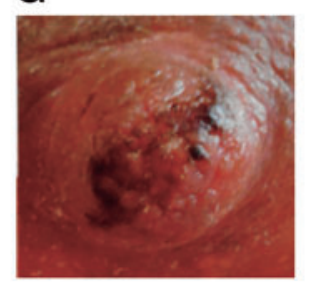

【Purpura】

K

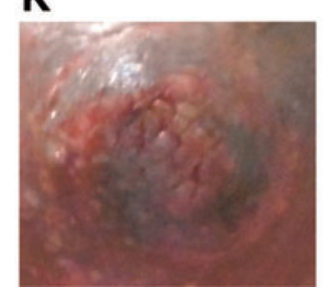

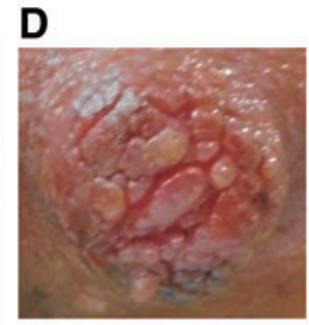

H

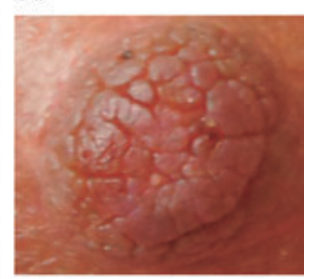

【Peeling】

L

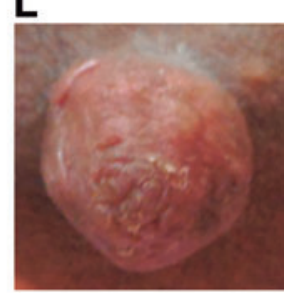

revealed that there were no significant differences in the incidences of each sign among participants regardless of parity, mode of delivery, or prior experience with breastfeeding.

Pain scores are shown in Table 2. The highest scores were associated with the appearance of scabbing. A comparison of pain scores, according to the presence or absence of each sign of trauma and small changes, showed that scores with signs of nipple trauma were significantly higher than those without signs of erythema, swelling, or scabbing.

\section{Phase 2}

Purpose

To examine the reliability of signs of nipple trauma identified in Phase 1.

\section{Materials and Methods}

Participants. The study participants included eight clinical midwives employed by the same obstetrics and gynecology facility. The average experience as a clinical midwife was

Table 1. Results of Observed Signs of Nipple Trauma and Other Changes

\begin{tabular}{|c|c|c|c|c|c|c|c|c|c|}
\hline \multirow[b]{2}{*}{$\begin{array}{l}\text { Postpartum } \\
\text { days }\end{array}$} & \multirow[b]{2}{*}{$\begin{array}{l}\text { Number } \\
\text { of images }\end{array}$} & \multicolumn{6}{|c|}{ Signs of nipple trauma } & \multicolumn{2}{|c|}{ Small changes } \\
\hline & & $\begin{array}{l}\text { Erythema } \\
\mathrm{n}(\%)\end{array}$ & $\begin{array}{l}\text { Swelling } \\
\mathrm{n}(\%)\end{array}$ & $\begin{array}{c}\text { Fissure } \\
\mathrm{n}(\%)\end{array}$ & $\begin{array}{c}\text { Blistering } \\
\mathrm{n}(\%)\end{array}$ & $\begin{array}{l}\text { Scabbing } \\
\mathrm{n}(\%)\end{array}$ & $\begin{array}{c}\text { Multiple signs } \\
\mathrm{n}(\%)\end{array}$ & $\begin{array}{c}\text { Purpura } \\
\text { n (\%) }\end{array}$ & $\begin{array}{l}\text { Peeling } \\
\mathrm{n}(\%)\end{array}$ \\
\hline Day 0 & 16 & $7(43.8)$ & $2(12.5)$ & $1(6.3)$ & $0(0.0)$ & $0(0.0)$ & 7 (43.8) & $1(6.3)$ & $0(0.0)$ \\
\hline Day 1 & 67 & $37(55.2)$ & $21(31.3)$ & $7(10.4)$ & $4(6.0)$ & $16(23.9)$ & $41(61.2)$ & $5(7.5)$ & $2(3.0)$ \\
\hline Day 2 & 122 & $81(66.4)$ & $45(36.9)$ & $21(17.2)$ & $4(3.3)$ & $36(29.5)$ & $96(78.7)$ & $9(7.4)$ & $5(4.1)$ \\
\hline Day 3 & 157 & $118(75.2)$ & $66(42.0)$ & $22(14.0)$ & $8(5.1)$ & $56(35.7)$ & $133(84.7)$ & $14(8.9)$ & $10(6.4)$ \\
\hline Day 4 & 166 & $114(68.7)$ & $78(47.0)$ & $23(13.9)$ & $9(5.4)$ & $68(41.0)$ & $130(78.3)$ & $14(8.4)$ & $10(6.0)$ \\
\hline Day 5 & 143 & $91(63.6)$ & 47 (32.9) & $18(12.6)$ & $1(0.7)$ & $58(40.6)$ & $104(72.7)$ & $12(8.4)$ & $8(5.6)$ \\
\hline Day 6 & 68 & 35 (51.5) & $17(25.0)$ & 13 (19.1) & $2(2.9)$ & $25(36.8)$ & 44 (64.7) & $5(7.4)$ & $2(2.9)$ \\
\hline Day 7 & 24 & $9(37.5)$ & $3(12.5)$ & $7(29.2)$ & $2(8.3)$ & $8(33.3)$ & $13(54.2)$ & $2(8.3)$ & $2(8.3)$ \\
\hline Day 8 & 8 & $4(50.0)$ & $1(12.5)$ & $0(0.0)$ & $0(0.0)$ & $4(50.0)$ & $5(62.5)$ & $2(25.0)$ & $2(25.0)$ \\
\hline Day 9 & 5 & $3(60.0)$ & $0(0.0)$ & $0(0.0)$ & $0(0.0)$ & $2(40.0)$ & $3(60.0)$ & $2(40.0)$ & $0(0.0)$ \\
\hline Total & 776 & 499 & 280 & 112 & 30 & 273 & 576 & 66 & 41 \\
\hline
\end{tabular}

Data are numbers of each sign observed and divided by the total number of images in each postpartum day. 
Table 2. Pain Scores by Signs of Nipple Trauma and Small Changes

\begin{tabular}{|c|c|c|c|c|c|c|c|c|c|}
\hline & & \multicolumn{3}{|c|}{ Present } & \multicolumn{3}{|c|}{ Absent } & \multirow{2}{*}{\multicolumn{2}{|c|}{$\begin{array}{c}\text { Mann-Whitney } \\
\text { U test }\end{array}$}} \\
\hline & & \multirow[b]{2}{*}{$\mathrm{n}$} & \multicolumn{2}{|c|}{ Pain } & \multirow[b]{2}{*}{$\mathrm{n}$} & \multicolumn{2}{|c|}{ Pain } & & \\
\hline & & & Mean $\pm S D$ & Median $(I Q R)$ & & Mean $\pm S D$ & Median (IQR) & $Z$ & $\mathrm{P}$ \\
\hline Signs of nipple & Erythema & 356 & $4.96 \pm 2.39$ & $5(3-7)$ & 120 & $4.03 \pm 2.58$ & $4(2-5)$ & -3.98 & 0.00 \\
\hline trauma & Swelling & 241 & $5.22 \pm 2.39$ & $5(3-7)$ & 235 & $4.22 \pm 2.45$ & $4(2-6)$ & -4.71 & 0.00 \\
\hline & Fissure & 83 & $4.80 \pm 2.05$ & $5(3-6)$ & 393 & $4.71 \pm 2.54$ & $4(3-7)$ & -0.81 & 0.42 \\
\hline & Blistering & 30 & $5.33 \pm 2.10$ & $5(3-7)$ & 446 & $4.69 \pm 2.49$ & $4(3-6)$ & -1.62 & 0.11 \\
\hline & Scabbing & 248 & $5.67 \pm 2.20$ & $6(4-7)$ & 228 & $3.67 \pm 2.31$ & $3(2-5)$ & -9.13 & 0.00 \\
\hline & Multiple signs & 407 & $5.05 \pm 2.43$ & $5(3-7)$ & 69 & $2.81 \pm 1.69$ & $3(1-4)$ & -7.21 & 0.00 \\
\hline Small changes & Purpura & 66 & $4.68 \pm 2.05$ & $5(3-6)$ & 410 & $4.17 \pm 2.35$ & $4(2-5)$ & -0.31 & 0.76 \\
\hline & Peeling & 31 & $4.42 \pm 2.70$ & $4(2-5)$ & 445 & $4.21 \pm 2.31$ & $4(2-5)$ & -0.58 & 0.57 \\
\hline
\end{tabular}

$N^{\mathrm{a}}=476$.

The Mann-Whitney U test was used to assess the relationship between nipple pain and the presence or absence of each sign of nipple trauma.

${ }^{\mathrm{a}}$ Total observation days of 50 participants were multiplied by two nipples.

$\mathrm{IQR}$, interquartile range; $\mathrm{SD}$, standard deviation.

$20.4 \pm 6.1$ years, which indicated their professional breastfeeding support experience. Two of them were International Board-Certified Lactation Consultants. Data were collected from July 2017 to August 2017.

Data collection. Midwives were shown 20 digital images obtained in Phase 1 and asked to assess the presence or absence of each sign of nipple trauma. Digital images of the signs of nipple trauma were randomly included. In addition, 2 of the 20 digital images were identical to evaluate intrarater reliability. To eliminate the influence of the order effect, images were presented in three sequences and participants selected one of the three sequences by lottery.

Data analysis. Kendall's coefficient of concordance and Cohen's kappa coefficient were calculated using R software (version 3.4.1) (The R Foundation for Statistical Computing, Vienna, Austria).

Ethics committee approval. The study protocol was approved by the Ethics Review Committee of the Graduate School of Health Sciences, Hokkaido University (Approval number: 17-45), and conducted in accordance with ethical principles of the Declaration of Helsinki for medical research involving human subjects.

\section{Results}

Table 3 shows inter- and intrarater reliability results for the five signs of nipple trauma. Kendall's coefficient of concordance, for inter-rater reliability, ranged from moderate to almost perfect $(w=0.46-0.85)$, with almost perfect agreement for fissure $(w=0.85)$, substantial agreement for blistering $(w=0.67)$ and scabbing $(w=0.60)$, and moderate agreement for erythema $(w=0.46)$ and swelling $(w=0.48)$. The agreement was good for erythema and swelling (both 75\%) and excellent for blistering, fissure, and scabbing (all 100\%).

The kappa values for intrarater reliability among the eight midwives were substantial to almost perfect $(w=0.54-1.00)$. The agreement rate was good to excellent in $80-100 \%$ of all results.

\section{Discussion}

This study described changes to nipple skin in breastfeeding mothers and classified five signs of nipple trauma. Among these signs, fissuring was observed only at the nipple-areolar

Table 3. The Inter- and Intrarater Reliability Results of Five Signs of NipPle Trauma

Inter-rater reliability

\begin{tabular}{lcccccc}
\hline Signs of nipple trauma & Erythema & Swelling & Blister & Fissure & Scabbing & All data \\
\hline Kendall's coefficient of concordance $(w)$ & 0.46 & 0.48 & 0.67 & 0.85 & 0.6 & 0.54 \\
Intrarater reliability agreement rate $(\%)^{\mathrm{a}}$ & 75 & 75 & 100 & 100 & 100 & 100 \\
\hline
\end{tabular}

Intrarater reliability

\begin{tabular}{|c|c|c|c|c|c|c|c|c|}
\hline Observer & $A$ & $B$ & $C$ & $D$ & $E$ & $F$ & $G$ & $H$ \\
\hline Cohen's kappa coefficient $(\kappa)$ & 1 & 0.54 & 0.61 & 0.61 & 1 & 1 & 0.61 & 1 \\
\hline Agreement rate $(\%)^{\mathrm{b}}$ & 100 & 80 & 80 & 80 & 100 & 100 & 80 & 100 \\
\hline
\end{tabular}

Agreement rate $(\%)=$ number of signs that matched judgment/five (number of identified nipple trauma signs) $\times 100$.

${ }^{\mathrm{a}}$ Agreement rate $(\%)=$ number of people whose judgment matched/number of participants $\times 100$.

${ }^{\mathrm{b}}$ The concordance rate at which one observer judged two identical images. 
junction, scabbing and blistering were classified as damages to the dermis, and erythema and swelling as damages to the epidermis. On the basis of the anatomical structure of the skin, scabbing and blistering were considered to be more serious signs of nipple trauma than erythema or swelling, and these findings were supported by the degree of nipple pain reported. When scabbing or blistering was observed, the mean value of nipple pain score was higher than that for erythema or swelling. As a result, scabbing and blistering can be used as indicators to assess the severity of nipple trauma.

Erythema, observed from day 0, was the most frequent observation in this study. These results were consistent with those of a previous study, which reported that normal breastfeeding induces an inflammatory response and results in substantial nipple skin damage in most breastfeeding women. ${ }^{12}$ As this previous study reported, erythema can also occur with normal lactation. However, considering continued lactation, the presence of erythema may be a risk factor for severe nipple trauma owing to skin fragility caused by inflammation. In addition, erythema was associated with more severe nipple trauma such as fissuring. Thus, erythema should be evaluated in terms of timing and the presence or absence of other signs.

According to the standard of Landis and Koch, ${ }^{13}$ reliabilities of these five signs of nipple trauma were confirmed in this study to be high, except for erythema and swelling. There are two possible explanations for moderate reliability of erythema. First is the difficulty of color assessment, with Ahlqvist et al. having reported a lower level of agreement with regard to skin color assessment in evaluation of the extensive use of peripheral venous catheters. ${ }^{14}$ Second, erythema may have been overlooked, particularly in cases where erythema was concomitant with more serious signs. With regard to swelling, the Japanese midwives may be unaware of this symptom because there is no expression in the Japanese language to describe the symptoms of swelling and edema in nipple trauma.

Because erythema and swelling were identified as the earliest signs of nipple trauma, as indicated by the highest frequencies of observations, preventive intervention of nipple trauma can be assessed by further analyzing the relationship between these signs and other factors, such as positioning, latch-on, lactation frequency, nipple morphology, and neonatal suckling. To this end, the ability to observe these signs is very important in clinical settings. Furthermore, because multiple signs can be observed on a single nipple, it is necessary to accurately record nipple trauma.

Scabbing itself is not necessarily a skin lesion, although the observation of scabbing is important to assess the healing process of nipple trauma. ${ }^{10}$ Therefore, scabbing that exceeds $20 \%$ of the nipple must be carefully evaluated on day 1 (Table 1). The most common signs of nipple trauma observed on day 0 were erythema and swelling, which indicated damage to the epidermis. Thus, there is a possibility that the signs of erythema and swelling observed on day 0 worsened and skin damage deepened because of continued lactation, even within 24 hours.

Purpura and peeling were small changes observed by close interpretation of photographs. ABM Protocol No. 26 has defined purpura as bruising of the nipple due to changes induced by misuse of a breast pump. ${ }^{15}$ Peeling is noted as changes in nipple trauma in some articles. ${ }^{6,7}$ Although these skin changes are often overlooked in clinical settings, physicians and midwives must consider mechanisms underlying the development of nipple trauma.

A limitation to this study was that data were collected from a single clinic. Some types of nipple trauma, such as ulceration, which indicate deterioration of skin conditions, were not observed in this study. Therefore, the possibility that the breastfeeding support provided in the clinic would be reflected in results cannot be overlooked. However, a strength of this study was the classification of the signs of nipple trauma in breastfeeding women during postpartum week 1, as verified with the cooperation of clinical midwives. Future studies are warranted to explain the healing process and to develop a scale to assess nipple trauma on the basis of our results. The development of effective methods for prevention and treatment of nipple trauma on the basis of our findings should prove useful in clinical settings.

\section{Conclusion}

Image analysis revealed five signs of nipple traumaerythema, swelling, blistering, fissure, and scabbing. Scabbing and blistering can be used as indicators to assess the severity of nipple trauma based on the mothers' subjective experiences of pain intensity. In addition, reliability of these signs for establishing nipple trauma was confirmed. Erythema and swelling were the most frequently observed signs in nipple skin trauma during postpartum week 1. However, the agreement rate for these signs was lower than that for other signs, suggesting the possibility of conflicting interpretations in clinical evaluation.

\section{Acknowledgments}

The authors wish to thank the mothers, midwives, and staff of the clinic, including the director, who cooperated in this research.

\section{Disclosure Statement}

The authors have no competing financial interests to declare. M.N., T.O., and Y.Y. recruited the women and M.N. collected data. M.N. and Y.A. analyzed the results.

\section{References}

1. Odom EC, Li R, Scanlon KS, et al. Reasons for earlier than desired cessation of breastfeeding. Pediatrics 2013;131: e726-e732.

2. Li R, Fein SB, Chen J, et al. Why mothers stop breastfeeding: Mothers' self-reported reasons for stopping during the first year. Pediatrics 2008;122:S69-S76.

3. Vieira F, Bachion MM, Mota DD, et al. A systematic review of the interventions for nipple trauma in breastfeeding mothers. J Nurs Scholarsh 2013;45:116-125.

4. Dennis CL, Schottle N, Hodnett E, et al. An all-purpose nipple ointment versus lanolin in treating painful damaged nipples in breastfeeding women: A randomized controlled trial. Breastfeed Med 2012;7:473-479.

5. Marrazzu A, Sanna MG, Dessole F, et al. Evaluation of the effectiveness of a silver-impregnated medical cap for topical treatment of nipple fissure of breastfeeding mothers. Breastfeed Med 2015;10:232-238. 
6. Henderson A, Stamp G, Pincombe J. Postpartum positioning and attachment education for increasing breastfeeding: A randomized trial. Birth 2001;28:236-242.

7. Brent N, Rudy SJ, Redd B, et al. Sore nipples in breastfeeding women: A clinical trial of wound dressings vs conventional care. Arch Pediatr Adolesc Med 1998;152: 1077-1082.

8. Morland-Schultz K, Hill PD. Prevention of and therapies for nipple pain: A systematic review. J Obstet Gynecol Neonatal Nurs 2005;34:428-437.

9. Dennis CL, Jackson K, Watson J. Interventions for treating painful nipples among breastfeeding women. Cochrane Database Syst Rev 2014:CD007366D

10. Cervellini MP. Injuries resulted from breastfeeding: A new approach to a known problem. Rev Esc Enferm USP 2014; 48:346-356 (Article in Portuguese).

11. Word Health Organization. Breastfeeding counselling a training course. 1993. Available at www.who.int/nutrition/ publications/infantfeeding/bf_counselling_participants_ manual1.pdf (accessed May 18, 2016).
12. Ziemer MM, Pigeon JG. Skin changes and pain in the nipple during the 1st week of lactation. J Obstet Gynecol Neonatal Nurs 1993;22:247-256.

13. Landis JR, Koch GG. The measurement of observer agreement for categorical data. Biometrics 1977;33:159-174.

14. Ahlqvist M, Berglund B, Nordstrom G, et al. A new reliable tool (PVC assess) for assessment of peripheral venous catheters. J Eval Clin Pract 2010;16:1108-1115.

15. Berens P, Eglash A, Malloy M, et al. ABM clinical protocol \#26: Persistent pain with breastfeeding. Breastfeed Med 2016;11:46-53.

Address correspondence to: Yoko Asaka, PhD

Faculty of Health Sciences

Hokkaido University

N12-W5, Kitaku Sapporo 060-0812

Japan

E-mail: y_asaka@hs.hokudai.ac.jp 